\title{
Review of: "Study on hyperspectral estimation model of soil organic carbon content in the wheat field under different water treatments"
}

Lixin Lin

Potential competing interests: The author(s) declared that no potential competing interests exist.

Many researchers have indicated that spectral transformations can help the hyperspectral estimation of SOC, and derivative analysis is commonly used. Two examples are the research by Vašát et al. [1] and Tsai and Philpot [2]. Vašát et al. used nine different spectra transforms and found the first order derivative produced the best performance. About the number of samples in calibration and validation, we agree the different proportion for calibration and validation can affect the SOC estimation accuracy like the research by Debaene et al. [3]. Based on the soil samples with different soil water treatments, this paper combined 17 kinds of spectral transformations with 5 allocation ratios of calibration to verification, and found the combination of first-order derivative with 3:2 calibration and validation proportion was the optimal scheme. This paper is interesting and significant for readers. But there is a considerable body of research in this field that was ignored by the authors, so I suggest the authors can discuss more about the findings of others in their "Introduction". The authors can refer to the following related papers.

[1] Vašát, R., Kodešová, R., Klement, Aleš., Borůvka, L., 2017. Simple but efficient signal pre-processing in soil organic carbon spectroscopic estimation. Geoderma 298, 46-53 (2017).

[2] Tsai, F., Philpot,W., 1998. Derivative analysis of hyperspectral data. Remote Sens. Environ. 66, 41-51.

[3] Debaene, G., Nied'zwiecki, J., Pecio, Z' urek, A., 2014. Effect of the number of calibration samples on the prediction of several soil properties at the farm-scale. Geoderma 214, 114-125. 